\title{
A Reforma Psiquiátrica Brasileira: perspectivas e problemas
}

\author{
Manoel Tosta Berlinck \\ Ana Cecília Magtaz \\ Mônica Teixeira
}

A Reforma Psiquiátrica Brasileira, ao engendrar novos dispositivos assistenciais e novas práticas clínicas, cria atividades práticas anteriormente desconhecidas da saúde mental. Este projeto de pesquisa pretende contribuir para o avanço do conhecimento dessas novas práticas clínicas. Para tanto, propõe a criação do Laboratório de Saúde Mental, um grupo permanente de pesquisa que reunirá trabalhadores de saúde mental para narrarem suas vivências clínicas. Pretende-se com isso: 1) construir um arquivo das vivências clínicas de trabalhadores de saúde mental; 2) transformar vivências clínicas em experiências socialmente compartilhadas; e 3) criar um banco de dados sobre as práticas clínicas no âmbito da Reforma Psiquiátrica Brasileira para uso de trabalhadores e pesquisadores.

Palavras-chave: Reforma Psiquiátrica Brasileira, saúde mental, prática clínica 
A Reforma Psiquiátrica Brasileira é um movimento sociopolítico ocorrendo no âmbito da saúde pública que, do ponto de vista da gestão de políticas públicas, consubstancia-se em uma legislação em saúde mental iniciada em 1990, com a Declaração de Caracas, aprovada por aclamação pela Conferência Regional para a Reestruturação da Assistência Psiquiátrica dentro dos Sistemas Locais de Saúde.

O Brasil é aderente a essa Declaração, e a ela se articula com um longo e conturbado movimento de trabalhadores de saúde mental que resultou na Lei n. 9.867, de 10 de novembro de 1999.

Tal lei permite o desenvolvimento de programas de suporte psicossocial para os pacientes psiquiátricos em acompanhamento nos serviços comunitários. É um valioso instrumento para viabilizar os programas de trabalho assistido e inclui-los na dinâmica da vida diária, em seus aspectos econômicos e sociais. Há uma evidente analogia com as chamadas "empresas sociais" da experiência da Reforma Psiquiátrica Italiana.

Em 6 de abril de 2001, o Governo Federal promulga a Lei n. 10.216 que dispõe sobre a proteção e os direitos das pessoas portadoras de transtornos mentais e redireciona o modelo assistencial em saúde mental. Esse texto reflete o consenso possível sobre uma lei nacional para a reforma psiquiátrica no Brasil.

Tem como base o projeto original do deputado Paulo Delgado e inclui proposições contidas em substitutivos anteriores favoráveis ao projeto original.

A lei redireciona o modelo da assistência psiquiátrica, regulamenta cuidado especial com a clientela internada por longos anos e prevê a possibilidade de punição para a internação voluntária arbitrária ou desnecessária.

Em 28 de maio de 2003, o Presidente da República assina decreto instituindo Grupo de Trabalho interministerial para avaliar e apresentar propostas para rever, propor e discutir a política do 
governo federal para a atenção a usuários de álcool, bem como harmonizar e aperfeiçoar a legislação que envolva o consumo e a propaganda de bebidas alcoólicas em território nacional.

No dia 31 de julho de 2003, o Presidente da República assina a Lei n. 10.708 instituindo o auxílio-reabilitação psicossocial para pacientes acometidos de transtornos mentais, egressos de internações. Essa lei, conhecida como "Lei do Programa de Volta para a Casa", estabelece um novo patamar na história do processo de reforma psiquiátrica brasileira, impulsionando a desinstitucionalização de pacientes com longo tempo de permanência em hospital psiquiátrico, pela concessão de auxílio-reabilitação psicossocial e inclusão em programas extrahospitalares de atenção em saúde mental.

Desde 1992, existem oito leis estaduais em vigor, inspiradas no Projeto de Lei Federal do Deputado Paulo Delgado. Elas são do Ceará, do Distrito Federal, do Espírito Santo, de Minas Gerais, do Paraná, de Pernambuco, do Rio Grande do Norte e do Rio Grande do Sul. Em todas elas está prevista a substituição progressiva da assistência no hospital psiquiátrico por outros dispositivos ou serviços. Há incentivos para os centros de atenção diária (Centros de Atenção Psicossocial - CAPS), a utilização de leitos em hospitais gerais, a notificação de internação involuntária e a definição dos direitos das pessoas com transtornos mentais.

Há, hoje, no Brasil, mais de 1.000 Centros de Atenção Psicossocial - CAPS regulamentados, em sua estrutura e funcionamento, pela Portaria do Ministério da Saúde n. 336, de 19 de fevereiro de 2002. Além disso, há uma série de Portarias do Ministério da Saúde. A principal é, sem sombra de dúvida, a Portaria Interministerial n. 628, de 2 de abril de 2002, que estabelece o Plano Nacional de Saúde no Sistema Penitenciário. Ela define mecanismos de extensão da assistência em saúde do Sistema Único de Saúde (SUS) aos detentos do sistema penitenciário, incluindo-se saúde mental. Essa iniciativa inédita permite não só estender o olhar e o cuidado em saúde mental às prisões, mas também pensar um novo modo de lidar com a questão dos manicômios judiciários.

É importante destacar também a Portaria n. 1.077, de 24 de agosto de 1999, que dispõe sobre a assistência farmacêutica na atenção psiquiátrica e assegura medicamentos básicos de saúde mental para usuários de serviços ambulatoriais públicos de saúde que disponham de atenção em saúde mental. Representa um aporte efetivo e regular de recursos financeiros para que os estados e municípios mantenham um programa de farmácia básica em saúde mental.

As demais Portarias visam aprimorar a Reforma Psiquiátrica Brasileira criando e regulamentando o financiamento dos Serviços Residenciais Terapêuticos, determinando avaliação da assistência prestada em saúde mental pelo SUS, 
estabelecendo mecanismos de supervisão continuada dos serviços hospitalares e ambulatoriais, bem como proposição de normas técnicas e alternativas que reforcem a continuidade dos processos de reversão do modelo de atenção em saúde mental vigente no país, definindo a equipe mínima dos serviços ambulatoriais especializados em saúde mental que deve atuar na assistência e supervisão das atividades dos Serviços Residenciais Terapêuticos, instituindo o processo sistemático e anual de avaliação e supervisão da rede hospitalar especializada em psiquiatria, assim como hospitais gerais com enfermarias ou leitos psiquiátricos, estabelecendo critérios de classificação conforme tamanho do estabelecimento e cumprimento dos requisitos qualitativos de avaliação fixados pela área técnica de Saúde Mental do Ministério da Saúde, quanto ao processo terapêutico, e Agência Nacional de Vigilância Sanitária - ANVISA, para análise da área de vigilância sanitária, estabelecendo classificação baseada no porte do hospital e na qualidade do atendimento prestado, e assim por diante.

Em resumo, o que se observa é que o período de 1990 a 2003 concentra a máxima intensidade política e normativa do que se chama a Reforma Psiquiátrica Brasileira. Um ano antes, em 1989, iniciara-se a experiência decisiva em Santos, sob a liderança de David Capistrano Filho. Também no final desse mesmo ano deu entrada no Congresso Nacional o projeto de lei que resultou, 12 anos depois, na lei brasileira de reforma psiquiátrica.

A Reforma Psiquiátrica Brasileira realiza, então, por meio de uma complexa e sistemática política pública (Public Policy), consubstanciada em leis e portarias ministeriais, leis estaduais e municipais, uma ampla modificação no atendimento ao usuário: redireciona o modelo da assistência psiquiátrica; regulamenta cuidado especial com a clientela internada por longos anos, e prevê possibilidade de punição para a internação involuntária ou desnecessária; impulsiona a desinstitucionalização de pacientes com longo tempo de permanência em hospital psiquiátrico, pela concessão de auxílio-reabilitação psicossocial e inclusão em programas extrahospitalares de atenção; inclui assistência de saúde mental para os detentos do sistema penitenciário, possibilitando o pensamento sobre um novo modo de tratar com a questão dos manicômios judiciários; dispõe sobre assistência farmacêutica na atenção psiquiátrica e assegura medicamentos básicos de saúde mental para usuários de serviços ambulatoriais públicos de saúde que disponham de atenção em saúde mental etc.

Em outras palavras, a Reforma formula, cria condições e institui novas práticas terapêuticas visando a inclusão do usuário em saúde mental na sociedade e na cultura.

O sucesso da Reforma depende, portanto, de novas formas de clinicar e praticar o tratamento, e supõe que o trabalhador em saúde mental esteja preparado 
para realizar essas atividades. Sabe-se, entretanto, que entre o código e a prática há uma distância que nem sempre se revela nos relatórios oficiais encaminhados, periódica e sistematicamente, ao Ministério da Saúde.

Além disso, sabe-se, também, que o ensino universitário brasileiro ainda não se adaptou a essa política pública havendo uma defasagem entre formação universitária e os requisitos advindos da prática. Finalmente, o sistema universitário tende a oferecer um ensino geral e abstrato que é rico de conteúdo, mas que ignora, em grande parte, as especificidades da Reforma Psiquiátrica e o trabalho que, nesse âmbito, vem sendo realizado.

Tendo engendrado novos dispositivos e novas práticas clínicas, a Reforma Psiquiátrica criou vivências - atividades eminentemente práticas - anteriormente desconhecidas da saúde mental. Entretanto, essas vivências correm o grave risco de se circunscreverem ao específico âmbito de sua prática criando uma subcultura na sociedade brasileira. Ocorreria, assim, um fracasso da própria Reforma, cujo principal objetivo é a integração desse sistema - e não apenas dos usuários - na sociedade democrática e cidadã que se caracteriza pelo livre intercâmbio biológico, psíquico e social.

Assim, a Reforma Psiquiátrica Brasileira precisa, também, criar um dispositivo para que tais vivências se transformem em experiências, ou seja, em saberes socialmente compartilhados, desenvolvendo capacidades de pesquisa em saúde mental. Para que isso ocorra é necessário que as vivências eminentemente práticas dos trabalhadores de saúde mental sejam narradas e registradas constituindo, dessa forma, um arquivo, parte fundamental da memória das práticas promovidas pela Reforma. Em outras palavras, é necessária a realização de um trabalho de autoria, junto aos trabalhadores de saúde mental, que implique um processo de transformação das vivências em experiências, passando pela narrativa escrita, pelo registro narrativo da prática.

Tal atividade requer um dispositivo apropriado e complexo. Assim como foram necessários novos dispositivos clínicos, é necessária, agora, a criação de um outro dispositivo que engendre a narrativa escrita de vivências clínicas, a criação de um arquivo e de divulgação dessas mesmas narrativas para que os trabalhadores se tornem autores da própria Reforma e a sociedade fique sabendo o que ocorre no seu âmbito.

Esse dispositivo vem sendo criado e implantado pela Associação Universitária de Pesquisa em Psicopatologia Fundamental (AUPPF), sociedade científica sem fins lucrativos que reúne, atualmente, 50 professores doutores de 23 universidades brasileiras e de universidades da Argentina, da Colômbia, do México e da França.

A AUPPF criou recentemente (2007) um Laboratório de Saúde Mental destinado a estimular a escrita de narrativas por trabalhadores de saúde mental 
junto com uma reflexão a respeito delas. Essas narrativas são arquivadas e publicadas no portal (site) da Associação:

http://www. fundamentalpsychopathology.org

Articuladas a essas atividades, a Revista Latinoamericana de Psicopatologia Fundamental possui uma seção destacada na área de Saúde Mental, desde março de 2004. Mais recentemente, o Latin-American Journal of Fundamental Psychopathology on Line também publica artigos de universitários e de trabalhadores de saúde mental sobre essas vivências clínicas, que surgiram graças à Reforma Psiquiátrica Brasileira.

Constrói-se, assim, um dispositivo de autoria, arquivo e divulgação de narrativas e de reflexões sobre as práticas ocorridas no âmbito da Reforma Psiquiátrica Brasileira que servirão de feedback para o Ministério da Saúde, para as Secretarias Estaduais de Saúde e para as Secretarias Municipais de Saúde do Brasil, os quais poderão se beneficiar visando o aperfeiçoamento da política pública em saúde mental.

A Associação Universitária de Pesquisa em Psicopatologia Fundamental pretende, também, com esse trabalho, fornecer espaço para o arquivo e a publicação de narrativas clínicas praticadas em outros países. Ela visa, dessa forma, o aprimoramento das práticas clínicas por meio da construção de uma experiência que seja internacionalmente compartilhada.

\section{Resumos}

(Laboratorio de Salud Mental: proyecto de investigación)

La Reforma Psiquiátrica Brasileña, al engendrar nuevos dispositivos asistenciales y nuevas prácticas clínicas, crea actividades prácticas de salud mental anteriormente desconocidas. Este proyecto de investigación se propone contribuir para el avance del conocimiento de esas nuevas prácticas clínicas. Para tanto, propone la creación del Laboratorio de Salud Mental, grupo permanente de investigación que reunirá trabajadores de salud mental para que narraren sus vivencias clínicas. Se busca con eso: 1) construir un archivo de vivencias clínicas de trabajadores de salud mental; 2) transformar vivencia clínicas en experiencias socialmente compartidas y, 3) crear un banco de datos sobre las prácticas clínicas en el ámbito de la Reforma Psiquiátrica Brasileña para uso de trabajadores e investigadores.

Palabras claves: Reforma Psiquiátrica Brasileña, salud mental, práctica clínica 
(Laboratoire de Santé Mentale: projet de recherche)

En produisant de nouveaux dispositifs de soins, ainsi que de nouvelles pratiques cliniques, la réforme psychiatrique brésilienne crée des activités pratiques préalablement inconnues dans le domaine de la santé mentale. Le but de ce projet de recherche est de contribuer au développement des connaissances de ces nouvelles pratiques cliniques. Il propose, à cette fin, la création du Laboratoire de Santé Mentale, un groupe permanent de recherche qui réunira les travailleurs de la santé mentale. Ceux-ci seront invités à faire le récit de leurs expériences cliniques afin de: 1) construire un archive des expériences cliniques des travailleurs de la santé mentale; 2) transformer les expériences cliniques en expériences socialement partagées et 3) créer une banque de données sur les pratiques cliniques dans le cadre de la Réforme Psychiatrique Brésilienne qui sera mise à disposition des travailleurs et des chercheurs.

Mots clés: Réforme Psychiatrique Brésilienne, santé mentale, pratique clinique

\section{(Mental Health Laboratory: Research project)}

The Brazilian psychiatric reform resulted in new ways of caring for patients and new clinical practices. In the process, it brought with it innovative practical activities in mental health. This research project is intended as a contribution to the advance of knowledge regarding these new clinical practices. For this purpose, the author suggests the establishment of a Mental Health Laboratory, that is, a permanent research group to bring together workers in mental health to describe and discuss their clinical experiences. The objectives of this project are to: 1) set up an archive of clinical experiences of workers in mental health; 2) Transform clinical experiences into socially shared experiences, and, 3) Set up a databank on clinical practices in the scope of the Brazilian Psychiatric Reform for use by workers and researchers.

Key words: Brazilian Psychiatric Reform, mental health, clinical practice

Versão inicial recebida em outubro de 2007 Versão aprovada para publicação em janeiro de 2008

\section{Manoel Tosta BerLinck}

Sociólogo; psicanalista; Ph.D. pela Cornell University; professor do Programa de Estudos PósGraduados em Psicologia Clínica da Pontifícia Universidade Católica de São Paulo - PUCSP, onde dirige o Laboratório de Psicopatologia Fundamental (São Paulo, SP, Brasil); presi- 
dente da Associação Universitária de Pesquisa em Psicopatologia Fundamental (São Paulo, SP, Brasil); coordenador do Laboratório de Saúde Mental da Associação Universitária de Pesquisa em Psicopatologia Fundamental (São Paulo, SP, Brasil); editor responsável de Pulsional Revista de Psicanálise e da Revista Latinoamericana de Psicopatologia Fundamental; diretor da Editora Escuta e da Livraria Pulsional - Centro de Psicanálise; autor de Psicopatologia fundamental (São Paulo: Escuta), entre outros.

Rua Tupi, 397/103

01233-001 São Paulo, SP, Brasil

Telefax: (011) 3825-8573

e-mail: mtberlin@uol.com.br

\section{Ana Cecília Magtaz}

Psicóloga; psicanalista; doutoranda no Laboratório de Psicopatologia Fundamental do Programa de Estudos Pós-Graduados em Psicologia Clínica da Pontifícia Universidade Católica de São Paulo - PUC-SP (São Paulo, SP, Brasil); professora do Curso de Psicopatologia da Faculdade de Saúde Pública da Universidade de São Paulo - USP (São Paulo, SP, Brasil); assistente administrativa da Associação Universitária de Pesquisa em Psicopatologia Fundamental (São Paulo, SP, Brasil).

Rua Prof. Pedro da Cunha, 65/31

05010-020 São Paulo, SP, Brasil

Fone: (11) 3675-0584

e-mail: acmscaz@uol.com.br

\section{Mônica Teixeira}

Jornalista na TV Cultura e coordenadora do Núcleo de Pesquisas e Desenvolvimento de Novas Tecnologias para a TV pública; psicanalista; coordenadora do Laboratório de Saúde Mental da Associação Universitária de Pesquisa em Psicopatologia Fundamental (São Paulo, SP, Brasil); organizadora do livro Universidade e governo: professores da Unicamp no periodo FHC (Escuta, 2003).

Av. Higienópolis, 318/13

01238-001 São Paulo, SP, Brasil

e-mail: armonica@uol.com.br 